\title{
Insulin Capture by an Insulin-linked Polymorphic Region G- quadruplex DNA Oligonucleotide
}

\author{
Adam C. Connor, \\ Department of Chemistry, P. M. Gross Chemical Laboratory, Duke University, Durham, NC 27708 \\ Kimberley A. Frederick, \\ Department of Chemistry, College of the Holy Cross, 1 College Street, Worcester, MA 01610 \\ Elizabeth J. Morgan, and \\ Department of Chemistry and Chemical Biology, 118 Cogswell, Rensselaer Polytechnic Institute, \\ Troy, NY 12180, Tel: (518) 276-3861, Fax: (518) 276-4887 \\ Linda B. McGown \\ Department of Chemistry and Chemical Biology, 118 Cogswell, Rensselaer Polytechnic Institute, \\ Troy, NY 12180, Tel: (518) 276-3861, Fax: (518) 276-4887, mcgowl@rpi.edu
}

\begin{abstract}
Insulin capture by a G-quadruplex DNA oligonucleotide containing a two-repeat sequence of the insulin-linked polymorphic region (ILPR) of the human insulin gene promoter region is reported. The immobilized oligonucleotide was demonstrated to capture human insulin from standard solutions and from nuclear extracts of pancreatic cells with high selectivity, using affinity MALDI-mass spectrometry and affinity capillary chromatography. Insulin was preferentially captured by the tworepeat ILPR oligonucleotide over another G-quadruplex forming oligonucleotide, the thrombin binding aptamer, as well as over a single repeat of the ILPR sequence that is not capable of forming the G-quadruplex architecture. Binding was shown to involve the beta chain of insulin, most likely through association with the two parallel loops of the G-quadruplex structure. The discovery raises the possibility that insulin may bind to G-quadruplex DNA formed in the ILPR in vivo and thereby play a role in modulation of insulin gene expression, and provides a basis for design of insulin analogs to probe this hypothesis. The availability of a DNA ligand to human insulin has analytical importance as well, offering an alternative to antibodies for in vitro or in vivo detection and sensing of insulin as well as its isolation and purification from biological samples.
\end{abstract}

\section{Introduction}

The insulin-linked polymorphic region (ILPR) is a non-coding minisatellite in the human insulin gene promoter region located 363 base pairs upstream of the insulin gene ${ }^{1-7}$. It is one of several genetic loci associated with genetic susceptibility to insulin dependent diabetes mellitus 8 . The ILPR contains tandem repeats of a G-rich repeat unit of the consensus sequence $5^{\prime}-\mathrm{ACAG}_{4} \mathrm{TGTG}_{4}-3^{\prime}$. Oligonucleotides containing at least two tandem repeats can form intramolecular G-quadruplex structures in vitro ${ }^{1}$. Polymorphism in the ILPR includes variability in the number of tandem repeats and variability in the repeat sequence arising from single base pair substitutions. Variants of the ILPR sequence that destabilize or preclude Gquadruplex formation are also associated with significantly lower transcription rates $1,8,9$, suggesting a possible role of G-quadruplex formation in the regulation of transcription the 
insulin gene. Supporting evidence is found in the case of the transcription factor Pur-1, which preferentially binds to ILPR tandem repeats that form inter- or intramolecular G-quadruplex structures. This has raised the hypothesis that Pur-1 modulates insulin transcription through recognition of G-quadruplex structures in the ILPR ${ }^{8}$; however, G-quadruplex recognition did not in all cases correlate with transcriptional activity, suggesting that there may be alternate regulatory mechanisms that involve other protein interactions with the "transcription machinery" or modifications of the ILPR tertiary structure ${ }^{8}$.

We report here the discovery that insulin exhibits binding affinity toward G-quadruplex DNA formed by a 2-repeat length of the ILPR consensus sequence (hereinafter referred to as " $I L \mathrm{LR}_{2}$ "). This intriguing finding raises the possibility that insulin itself may play a role in regulation of its own gene through association with G-quadruplexes formed in the ILPR region. Affinity capture of insulin from commercial protein samples, including purified human insulin and nuclear extracts from human pancreatic cells, by the ILPR 2 oligonucleotide was demonstrated using previously described platforms for affinity Matrix Assisted Laser Desorption Ionization-Mass Spectrometry (MALDI-MS) ${ }^{10}$ and affinity capillary chromatography ${ }^{11}$. The DNA oligonucleotides were immobilized through a covalent linker to fused silica surfaces. Immobilization is intrinsic to both capture schemes and provides a realistic model for protein binding to G-quadruplexes formed in the ILPR that would be anchored in the chromosomal strand. G-quadruplex formation by similarly immobilized DNA has been confirmed in separate studies of the thrombin-binding DNA aptamer ${ }^{10,11}$, which forms a 2-plane G-quadruplex structure ${ }^{12,13}$.

Results for ILPR 2 were compared with those for bare (unmodified) fused silica surfaces to account for any non-specific surface adsorption, surfaces coated with a single ILPR sequence ("ILPR 1 ") that cannot form an intramolecular G-quadruplex, in order to see if binding is due to sequence rather than conformation, surfaces coated with the thrombin-binding aptamer ("TBA") to test for specificity among different G-quadruplex DNA oligonucleotides, and surfaces coated with an oligonucleotide derived by scrambling the TBA sequence ("Scrambled") that cannot form a G-quadruplex 11 , in order to test for non-specific DNA binding. Cross reactivity among G-quadruplex DNA-binding proteins and G-quadruplex DNA was investigated by comparing capture of insulin and thrombin at ILPR 2 and TBA coated surfaces.

\section{Experimental}

\section{Materials}

The 5'-thiol modified oligonucleotides, including the G-quadruplex forming, double repeat ILPR sequence $\left(\mathrm{ILPR}_{2}\right)\left(5^{\prime}-\left(\mathrm{ACAG}_{4} \mathrm{TGTG}_{4}\right)_{2}-3^{\prime}\right)$, the ILPR single repeat sequence $\left(\mathrm{ILPR}_{1}\right)$ (5'-( $\left.\mathrm{ACAG}_{4} \mathrm{TGTG}_{4}-3^{\prime}\right)$, the thrombin-binding aptamer (TBA) (5'-

GGTTGGTGTGGTTGG-3'), and the scrambled TBA sequence (Scrambled) (5'-

GGTGGTGGTTGTGGT-3'), were custom synthesized by Eurogentech North America (San Diego, CA). The oligonucleotides were brought to a concentration of $2.5 \mathrm{mM}$ in deionized water and stored at $-4{ }^{\circ} \mathrm{C}$. Potassium monohydrogen phosphate, potassium dihydrogen phosphate, Trizma buffer and 3-aminopropyltriethoxysilane (3-APTES) were from Sigma (St. Louis, MO). Sulphosuccinimidyl 4-( $N$-maleimidomethyl)cylcohexane-1-carboxylate (SMCC) and tris(2-carboxyethyl)phosphine (TCEP) were from either Pierce Chemical (Rockford, IL) or Sigma. The MALDI matrix was either sinnapinic acid (SA), $20 \mathrm{mg} / \mathrm{ml}$ in 50:50 acetonitrile:water containing $0.1 \%$ trifluoroacetic acid, or 2-(4-hydroxyphenylazo)benzoic acid (HABA), $2.5 \mathrm{mg} / \mathrm{mL}$ in 50:50 acetonitrile:water, both from Fluka. Human insulin (10 mg/ $\mathrm{ml}$ in $25 \mathrm{mM}$ HEPES, $\mathrm{pH}$ 8.2) and human serum albumin (HSA), lyophilized powder, from Sigma, were diluted to the appropriate concentration with buffer. Thrombin $(156 \mu \mathrm{M}$ in $25 \mathrm{mM}$ HEPES) from Haematologic Technologies (Essex Junction, VT), Human pancreatic nuclear 
extract (HPNE) from Active Motif (Carlsbad, CA), and DL-1,4-Dithriothreitol 99\% (DTT) from Acros were used as received without further purification.

\section{Preparation of DNA-coated MALDI surfaces and capillaries}

Oligonucleotide-coated spots were prepared on fused silica slides of $1 \mathrm{~mm}$ thickness (Valley Design Co., Westford, MA), as previously described ${ }^{10}$. Briefly, this entailed activation of the fused silica surface by rinsing with methanol, water, and $1 \mathrm{M}$ sodium hydroxide, followed by attachment of 3-APTES and coating of the silated surface with the heterobifunctional linker to form spots of approximately $2 \mathrm{~mm}$ diameter. The 5'-thiol modified oligonucleotides were treated with TCEP to cleave the disulfide bonds and then reacted with the linker on the slide surface overnight at $4{ }^{\circ} \mathrm{C}$ to create the oligonucleotide-coated spots. The slides were then rinsed with $\mathrm{pH} 8$ potassium phosphate buffer to remove any unbound oligonucleotides, dried under nitrogen, sealed, and stored at room temperature.

For the affinity capillary chromatography experiments, the inner surfaces of bare fused-silica capillaries (Polymicro Technologies, Phoenix, AZ, USA) of $47 \mathrm{~cm}$ total length and $100 \mu \mathrm{m}$ inner diameter (I.D.) were coated with DNA using the same procedure described above for the MALDI plates ${ }^{10,11}$. Following the attachment process, the capillaries were flushed with Tris buffer $(20 \mathrm{mM}$ Tris, $5 \mathrm{mM} \mathrm{KCl}, \mathrm{pH} 7.3)$, sealed with parafilm and stored at $4{ }^{\circ} \mathrm{C}$.

\section{Protein capture experiments}

For the affinity MALDI experiments, $1 \mu \mathrm{l}$ of sample was incubated on each oligonucleotidecoated spot at room temperature for $30 \mathrm{~min}$. The slide was then rinsed with water to remove any unbound or weakly associated species, and dried. MALDI matrix was then added to the spots and allowed to crystallize. The slide was then mounted directly onto a conventional stainless steel MALDI target plate using double-sided tape and the spots were analyzed by MALDI-TOF-MS using either an Applied Biosystems Voyager DE or Bruker AutoFlex II system.

The affinity capillary chromatography experiments were performed using either a Beckman Coulter P/ACE 5000 or P/ACE MDQ CE system according to the following procedure: One capillary volume of protein was loaded onto the capillary under low pressure $(0.5 \mathrm{psi})$ and incubated overnight. This volume was followed by a capillary volume of wash buffer $(20 \mathrm{mM}$ Tris, $5 \mathrm{mM} \mathrm{KCl}$, pH 7.3), both of which were collected. This served as the "load" collection. Three more capillary volumes of the wash buffer were passed through the capillary under low pressure to remove loosely associated proteins from the surface and collected. This served as the "wash" collection. A single capillary volume of elute buffer (20 mM Tris, $8 \mathrm{M}$ urea) was then introduced and the capillary temperature, which was maintained at $25^{\circ} \mathrm{C}$ up to this point, was raised to $50^{\circ} \mathrm{C}$. After $30 \mathrm{~min}$, the capillary volume and two additional capillary volumes of the elute buffer were passed through the capillary, collected and combined to form the "elute" collection. Each of the collections, load, wash and elute, was diluted to a constant volume of $25 \mu \mathrm{l}$ using its corresponding wash or elute buffer and stored at $4{ }^{\circ} \mathrm{C}$.

In previous work, we showed that capillaries coated with TBA have a binding capacity of approximately 0.08 thrombin molecules per $\mathrm{nm}^{2}$ surface area ${ }^{11}$. Extending these results to the present work, as a first approximation, we could predict a total capacity of $60 \mathrm{pmol}$ protein for the capillaries of length and internal diameter that were used. This corresponds to an insulin concentration of $20 \mu \mathrm{M}$ in a single capillary volume of $3.7 \mu \mathrm{l}$. In order to ensure an excess of protein to maximize protein capture, a solution of ten times this concentration, $200 \mu \mathrm{M}$, of protein was incubated in the various capillaries. 
Total protein in each collected fraction was determined using fluorescence emission spectroscopy. Fluorescence emission spectra of collections from the capillaries and of human insulin standard solutions were collected using an SLM-AMINCO model 8100 Series 2 fluorescence spectrometer with an excitation wavelength of $280 \mathrm{~nm}$. Samples were contained in 0.1-cm pathlength quartz cuvets from Starna Cells. Concentrations were determined from calibration curves constructed using the standard solutions.

The identities of the proteins in the elute collections were investigated by conventional MALDI-TOF-MS. Prior to the analysis, the $25 \mu \mathrm{l}$ collection solutions were desalted and preconcentrated by passing the solution through a C-18 Zip-Tip reverse phase chromatography tip (Millipore). The retained proteins were eluted from the Zip-Tip with $5 \mu$ of either 50:50 acetonitrile $/ \mathrm{H}_{2} 0$ with $0.1 \%$ trifluoroacetic acid or sinnapinic acid matrix. In cases where $\mathrm{HABA}$ matrix was used, $1 \mu \mathrm{l}$ of the eluent was mixed with $1 \mu \mathrm{l}$ of HABA matrix on a stainless steel MALDI plate. Because SA matrix is recommended for masses up to 40,000 Da by the instrument manufacturer and HABA is most efficient for larger masses, both matrices were needed for complete protein analysis.

\section{Results and Discussion}

\section{Affinity Capture Studies}

Insulin Standard-The first set of experiments compared insulin capture at ILPR $\mathrm{R}_{2}$-coated surfaces with capture at other surfaces. Figure 1a shows the spectrum of insulin on a bare (uncoated) spot that was not rinsed prior to application of MALDI matrix. This spectrum exhibits the expected insulin peak at $5808 \mathrm{~m} / \mathrm{z}$ and serves as standard for comparison with spectra of the other surfaces. Figure $1 \mathrm{~b}$ shows the spectrum for insulin on a bare spot that was rinsed prior to application of matrix. The absence of any significant peaks confirms that protein is effectively removed from the bare surface in the rinsing step. Figure 1c shows the spectrum of insulin captured at the ILPR 2 surface according to the procedure described in the experimental section. Insulin capture is clearly indicated by the presence of peaks at $5808 \mathrm{~m} /$ $\mathrm{z}$ and $2900 \mathrm{~m} / \mathrm{z}$, corresponding to singly and doubly charged insulin ion, respectively. By comparison, very little insulin capture is indicated at the ILPR 1 surface (Figure 1d) or the scrambled TBA surface (Figure 1e), neither of which can form an intramolecular G-quadruplex structure. Specificity of insulin for the ILPR G-quadruplex over the TBA G-quadruplex is indicated by the much smaller degree of capture at the TBA surface (Figure 1f).

Table 1 shows quantitative results for insulin capture experiments from $10 \mu \mathrm{M}$ and $1 \mu \mathrm{M}$ insulin standard solutions. At both concentrations, insulin capture was at least 10 -fold greater for $\mathrm{ILPR}_{2}$ than any of the other surfaces (discounting the bare unrinsed surface). The standard deviations in all cases are much lower for the ILPR 2 surface than for the other surfaces, indicating that retention of insulin due to affinity capture at the $\mathrm{ILPR}_{2}$ surface is much more reproducible than retention due to non-specific adsorption events at the other surfaces.

Cross-reactivity between G-quadruplex-binding proteins and G-quadruplex DNA was further investigated using the affinity capillary chromatography method to compare capture of insulin with capture of thrombin on ILPR 2 -coated and TBA-coated capillaries. Protein identities were confirmed using MALDI-TOF-MS. Circular dichroism spectra (shown in supplementary material for insulin and ILPR 2 ) confirmed unfolding of the protein and DNA structures under the conditions used for release and elution of the captured protein. Fluorescence spectra of the elute collections, shown in Figure 2, were used to compare protein capture.

Based on the fluorescence spectral peak areas, we found that 5 times more insulin is captured on the ILPR 2 capillary as on the TBA capillary, and at least 3 times more thrombin is captured 
on the TBA capillary as on the ILPR 2 capillary. These results confirm that there is selectivity among the different G-quadruplex structures towards their respective protein targets.

Interestingly, the TBA and Scrambled capillaries gave similar results for insulin capture, indicating that the G-quadruplex aptamer is no better at capturing insulin than the scrambled, non-G-quadruplex oligonucleotide. The affinity of insulin for ILPR 2 but not for TBA is attributed to differences between the TBA structure ${ }^{13}$ and the proposed ILPR 2 intramolecular G-quadruplex structure ${ }^{1}$. The presence of four G-tetrads in $\mathrm{ILPR}_{2}$ vs. two in TBA is unlikely to be responsible since proteins generally interact with the connecting loops, rather than the G-tetrads themselves, which merely provide a scaffold for arrangement of the loops. Both Gquadruplex structures have two parallel loops on one end and a single, perpendicularly oriented loop on the other. In both, the loop on the single end has sequence -TGT-. The parallel loops have sequence -ACA- in ILPR 2 , compared to -TT- in TBA. It is known that the parallel loops in TBA are the site of interaction with thrombin ${ }^{14}$, and it is reasonable to infer that the parallel loops are the site of interaction between insulin and ILPR 2 , providing the structural basis for the differential interactions between the proteins and G-quadruplex structures.

Insulin and human serum albumin-Non-specific protein interference was evaluated using human serum albumin (HSA). In the affinity MALDI experiments, both pure HSA and equimolar mixtures of HSA and insulin were loaded onto ILPR coated plates. There was no evidence of significant capture of HSA at the ILPR spot, nor did HSA interfere with capture of insulin from an equimolar mixture of the two. Similarly, in the affinity capillary chromatography experiments, there was no evidence of HSA capture in the ILPR 2 -coated capillary, nor did the presence of equimolar concentrations of HSA interfere with the recovery of insulin.

Human Pancreatic Nuclear Extract-Insulin capture from commercial human pancreatic nuclear extract (HPNE) was investigated using both affinity MALDI-MS and affinity capillary chromatography. The pancreas was chosen as the source because it is the site of human insulin synthesis, and a nuclear extract was chosen instead of a total cell lysate because the nucleus is the site of genomic DNA localization within the cell.

Representative affinity MALDI-MS results are shown in Figure 3 for HPNE diluted 1:10 in SA matrix at a bare surface that was not rinsed prior to MALDI-MS analysis, and for capture at ILPR 2 and ILPR 1 surfaces. The spectrum of HPNE at the bare, unrinsed surface (Figure 3a) shows a number of peaks resulting from multiple proteins, as expected for a nuclear extract. The spectrum for ILPR 2 (Figure $3 \mathrm{~b}$ ) shows a large peak near $3400 \mathrm{~m} / \mathrm{z}$, tentatively assigned to the beta chain of insulin. Human insulin consists of a 21 amino acid alpha chain (2382 Da) and a 30 amino acid beta chain $(3430 \mathrm{Da})$ that are linked by two disulfide bonds. The presence of the individual chain is attributed to the presence in the commercial HPNE preparation of $500 \mu \mathrm{M}$ DTT, which can break the disulphide bonds between the alpha and beta chains in the insulin molecule. No peaks are evident in the spectrum for the ILPR 1 surface (Figure 3c), indicating no protein capture.

Insulin treated with DTT-In order to further investigate our hypothesis that the beta chain of insulin is the site for interaction with the ILPR 2 oligonucleotide, as indicated by the HPNE capture results, we conducted both affinity capillary chromatography and affinity MALDI capture experiments on insulin standard that was treated with DTT before capture. Figure 4 shows the mass spectra of the load, wash and elute collections for the affinity capillary chromatography capture experiment on an ILPR 2 coated capillary.

In the load collection, which contains unretained proteins in the sample that was incubated in the capillary overnight, we see approximately equal signals from both the alpha chain around 
$2380 \mathrm{~m} / \mathrm{z}$ and the beta chain around $3430 \mathrm{~m} / \mathrm{z}$. Peaks at higher $\mathrm{m} / \mathrm{z}$ correspond to multimers of the alpha and beta chains. In the wash collection, which contains loosely associated compounds removed during the subsequent washings of the capillary with three capillary volumes of buffer, a much larger peak is observed for the alpha chain than the beta chain. Finally, the elute collection, which contains affinity captured proteins that are released under the denaturing conditions of the elute buffer, exhibits a much larger peak for the beta chain than the alpha chain. This indicates that there is preferential retention of the beta chain over the alpha chain due to affinity interactions between the ILPR 2 G-quadruplex and the beta chain.

Affinity MALDI experiments of insulin treated with DTT provide additional support of our hypothesis that the beta chain is the site of interaction of insulin with the ILPR 2 G-quadruplex. Eleven different ILPR 2 spots incubated with DTT-treated insulin all exhibited capture of the beta chain while only four spots showed evidence of alpha chain capture. Averaged over the 11 spots, the intensity for the beta chain was $20,000 \pm 10,000(\mathrm{RSD}=50 \%)$ while that for the alpha chain was 4,000 $\pm 5,000$ ( $\mathrm{RSD}=125 \%$ ), which is insignificant. When the same sample was incubated at six ILPR 1 -coated spots, less than a tenth as much beta chain (mean intensity $=1,500 \pm 700, \mathrm{RSD}=47 \%$ ), and no alpha chain, was captured. These results show highly preferential capture of beta chain over alpha chain by ILPR 2 and much higher affinity of beta chain for ILPR 2 over ILPR 1 .

The implication of the insulin beta chain in the interaction between insulin and the ILPR 2 is particularly important because it opens the door to consideration of other insulin proteins, such as the insulin-like growth factors (IGFs), that contain the same alpha and beta chains as insulin in a contiguous peptide. The three-dimensional structures of the insulin proteins are similar, with identical hydrophobic cores 15,16 . Due to their structural similarity, the receptors of one protein can also bind to the others. Accumulation of insulin and IGFs in the nucleus, where they can participate directly in regulation of nuclear events, has been well described ${ }^{17-27}$. Like insulin, IGFs are circulating hormones that participate in many functions, including cell growth and proliferation, and have been implicated directly or indirectly in diabetes.

\section{Conclusion}

Affinity capture of insulin by the G-quadruplex formed by a two-repeat ILPR sequence is demonstrated. Only a small fraction as much insulin is captured by surfaces coated with the G-quadruplex thrombin-binding aptamer or with non-G-quadruplex forming oligonucleotides, including a single repeat of the ILPR consensus sequence or the scrambled sequence of the thrombin-binding aptamer. Cross-reactivity studies between the thrombin-binding aptamer and the two-repeat ILPR demonstrated specificity among G-quadruplex structures and their protein targets. Non-specific binding of human serum albumin by the two-repeat ILPR was negligible and albumin did not interfere with insulin capture. Binding of insulin to the two-repeat ILPR occurs through the beta-chain of insulin, most likely through interactions with the parallel loops of the intramolecular G-quadruplex.

The results of this work suggest that insulin proteins may participate in regulation of the insulin gene by association through the beta chain with intramolecular G-quadruplex structures in the ILPR region. Further studies are needed to test this hypothesis in vivo using insulin beta chain analogs and using molecular modeling to probe the nature of the molecular interaction. In the analytical arena, the availability of a DNA binding ligand to human insulin offers an alternative to antibodies for in vitro and in vivo detection and sensing of insulin as well as its isolation and purification from biological samples. Although the genome-inspired ILPR 2 binding ligand to insulin was not derived combinatorially and is not therefore an "aptamer", it offers the same advantages such as ease of production and manipulation, stability, relatively small size, and re-usability 28,29 . 


\section{Supplementary Material}

Refer to Web version on PubMed Central for supplementary material.

\section{Acknowledgements}

This material is based upon work supported by the National Institutes of Health (Grants 1R03 AG21742-01 and 1 R21 DK70762-01).

\section{References}

1. Catasti P, Chen X, Moyzis RK, Bradbury EM, Gupta G. J Mol Biol 1996;264:534. [PubMed: 8969303]

2. Bell GI, Karam JH, Rutter WJ. Proc Natl Acad Sci USA 1981;78:5759. [PubMed: 6272317]

3. Bell GI, Selby MJ, Rutter WJ. Nature 1982;295:31. [PubMed: 7035959]

4. Rotwein P, Yokoyama S, Didier DK, Chirgwin JM. Am J Hum Genet 1986;39:291. [PubMed: 2876625]

5. Hammond-Kosack MC, Kilpatrick MW, Docherty K. J Mol Endocrin 1992;9:221.

6. Hammond-Kosack MC, Docherty K. FEBS Lett 1992;301:79. [PubMed: 1451791]

7. Lew A, Rutter WJ, Kennedy GC. Proc Natl Acad Sci 2000;97:12508. [PubMed: 11070077]

8. Hammond-Kosack MC, Dobrinski B, Lurz R, Docherty K, Kilpatrick MW. Nucleic Acids Res 1992;20:231. [PubMed: 1741248]

9. Paquette J, Giannoukakis N, Polychronakos C, Vafiadis P, Deal C. J Biol Chem 1998;273:14164.

10. Dick LW, McGown LB. Anal Chem 2004;76:3037. [PubMed: 15167780]

11. Connor AC, McGown LB. J Chromatgr A. in press (available on-line)

12. Bock LC, Griffin LC, Latham JA, Vermaas EH, Toole JJ. Nature 1992;355:564. [PubMed: 1741036]

13. Kelly JA, Feigon J, Yeates TO. J Mol Biol 1996;256:417. [PubMed: 8604127]

14. Tasset DM, Kubik MF, Steiner W. J Mol Biol 1997;272:688. [PubMed: 9368651]

15. Rinderknecht E, Humbel RE. J Biol Chem 1978;253:2769. [PubMed: 632300]

16. Blundell TL, Bedarkar S, Humbel RE. Fed Proc 1983;42:2592. [PubMed: 6189745]

17. Smith RM, Jarett L. Proc Natl Acad Sci USA 1987;84:459. [PubMed: 3540967]

18. Heyner S, Rao LV, Jarett L, Smith RM. Dev Biol 1989;134:48. [PubMed: 2471664]

19. Soler AP, Thompson KA, Smith RM, Jarett L. Proc Natl Acad Sci USA 1989;86:6640. [PubMed: 2672001]

20. Dodson G, Steiner D. Current Opinion in Structural Biology 1998;8:189. [PubMed: 9631292]

21. Harada S, Loten EG, Smith RM, Jarett L. J Cell Physiol 1992;153:607. [PubMed: 1447321]

22. Harada S, Smith RM, Jarett L. Cell Biochem Biophys 1999;31:307. [PubMed: 10736753]

23. Lin YJ, Harada S, Loten EG, Smith RM, Jarett L. Proc Natl Acad Sci USA 1992;89:9691. [PubMed: 1409684]

24. Burwen SJ, Jones AL. Trends Biochem Sci 1987;12:159.

25. Smith RM, Harada S, Jarett L. Int Rev Cytol 1997;173:243. [PubMed: 9127955]

26. Jans DA, Hassan G. Bioessays 1998;20:400. [PubMed: 9670813]

27. Tsai WC, Bhattacharyya N, Han LY, Hanover JA, Rechler MM. Endocrinology 2003;144:5615. [PubMed: 14500580]

28. McGown LB, Joseph MJ, Pitner JB, Vonk GP, Linn CP. Anal Chem 1995;67:663A.

29. Mukhopadhyay R. Anal Chem A-Pages 2005;77:114A. 

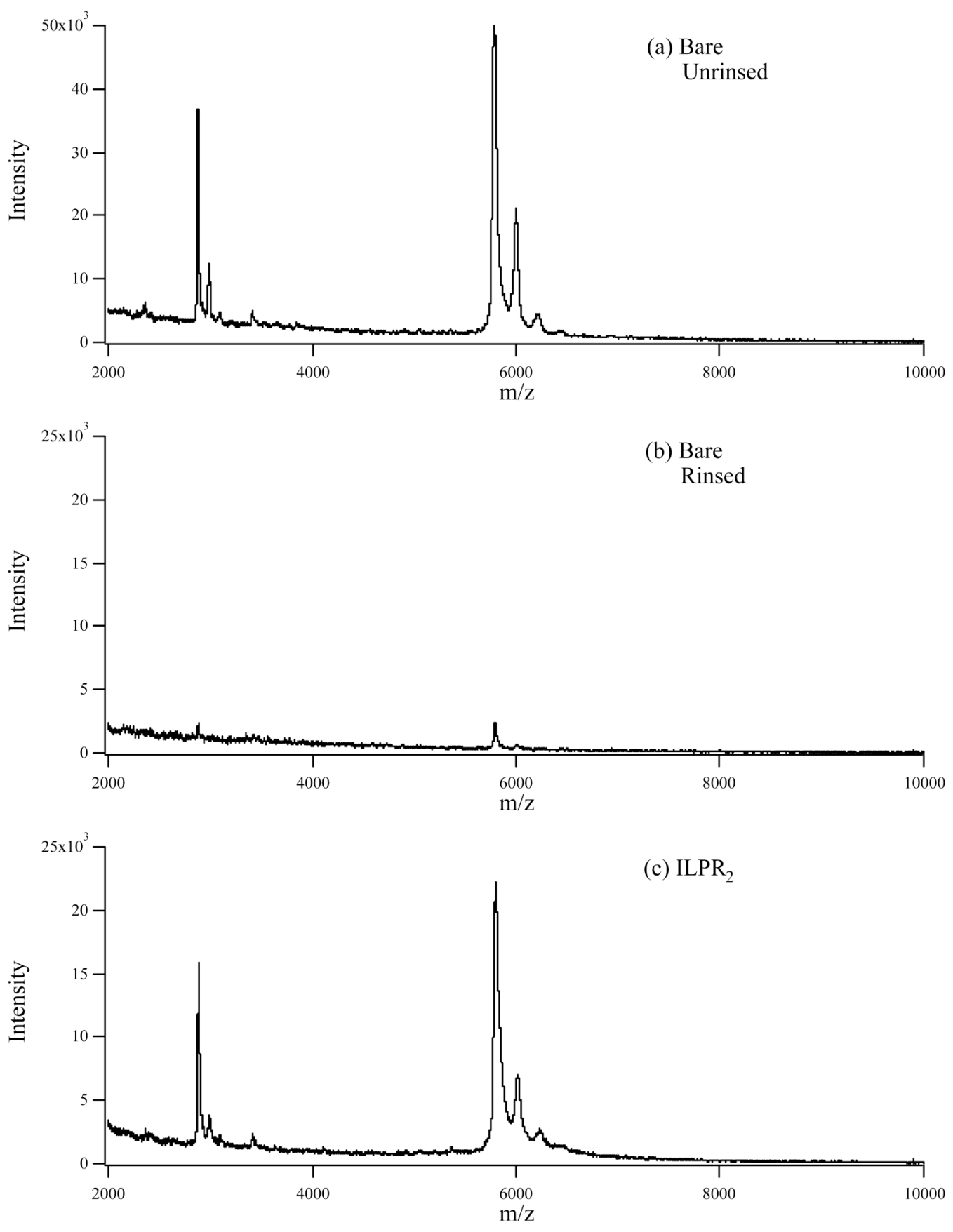

$J$ Am Chem Soc. Author manuscript; available in PMC 2008 September 30. 

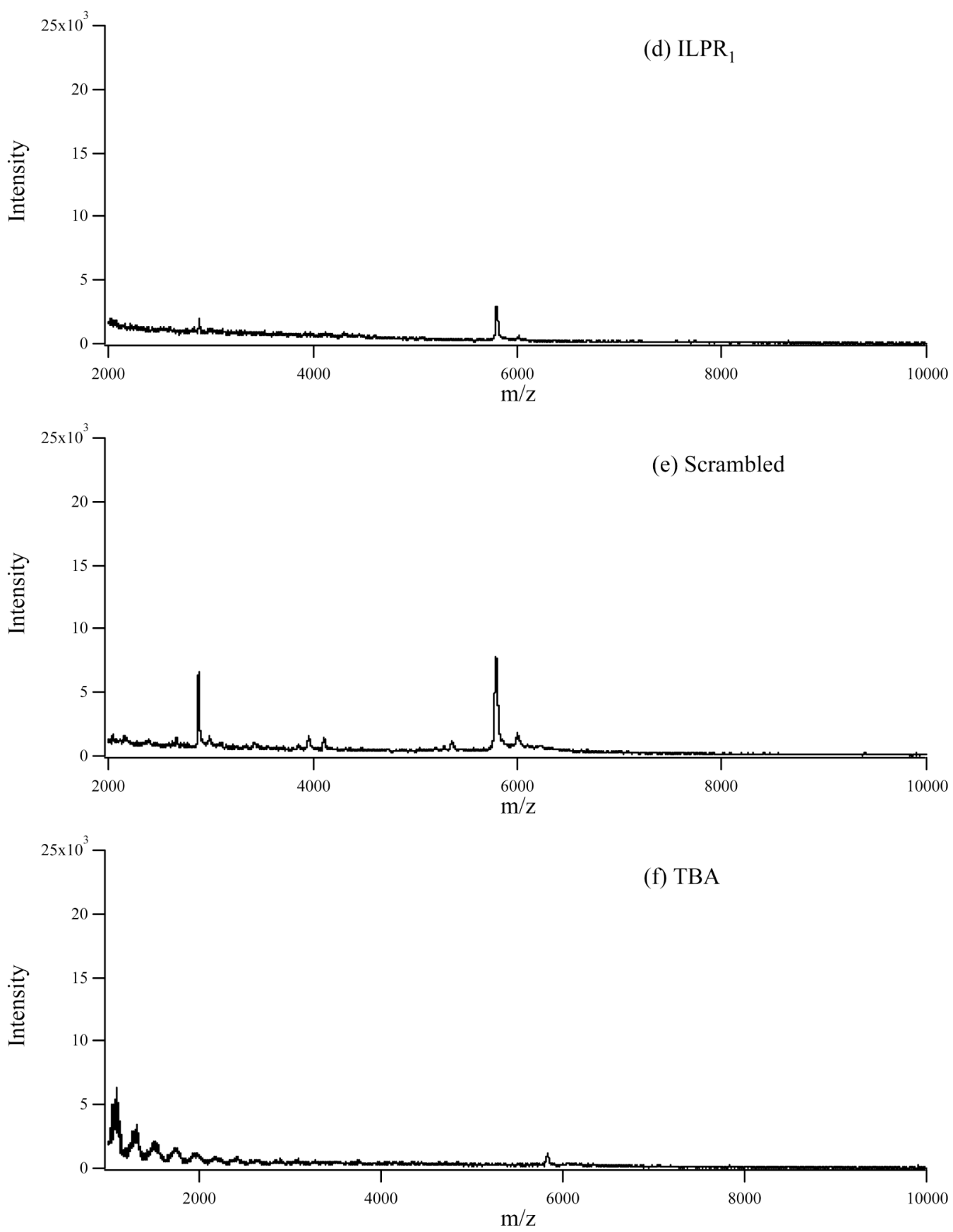

Figure 1.

MALDI-MS of $10 \mu \mathrm{M}$ insulin on various surfaces: (a) bare fused silica, unrinsed, (b) bare fused silica, rinsed, (c) ILPR 2 , (d) ILPR 1 , (e) Scrambled, and (f) TBA. 


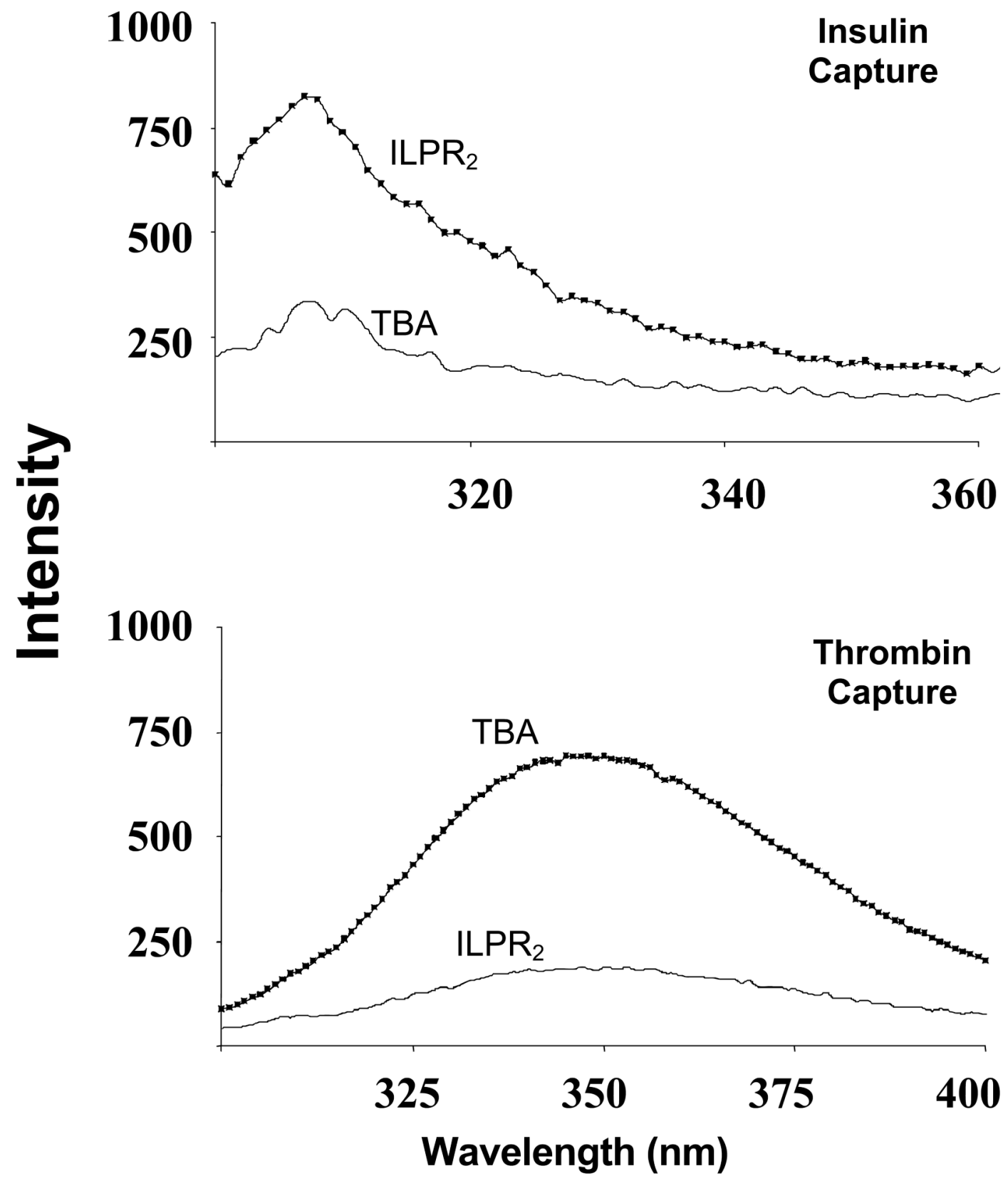

Figure 2.

Fluorescence emission spectra of collections of captured proteins. Top: Insulin captured from 1 capillary volume of $200 \mu \mathrm{M}$ insulin and eluted from ILPR 2 and TBA coated capillaries. Bottom: Thrombin captured from 1 capillary volume of $156 \mu \mathrm{M}$ thrombin and eluted from TBA and ILPR 2 coated capillaries. 

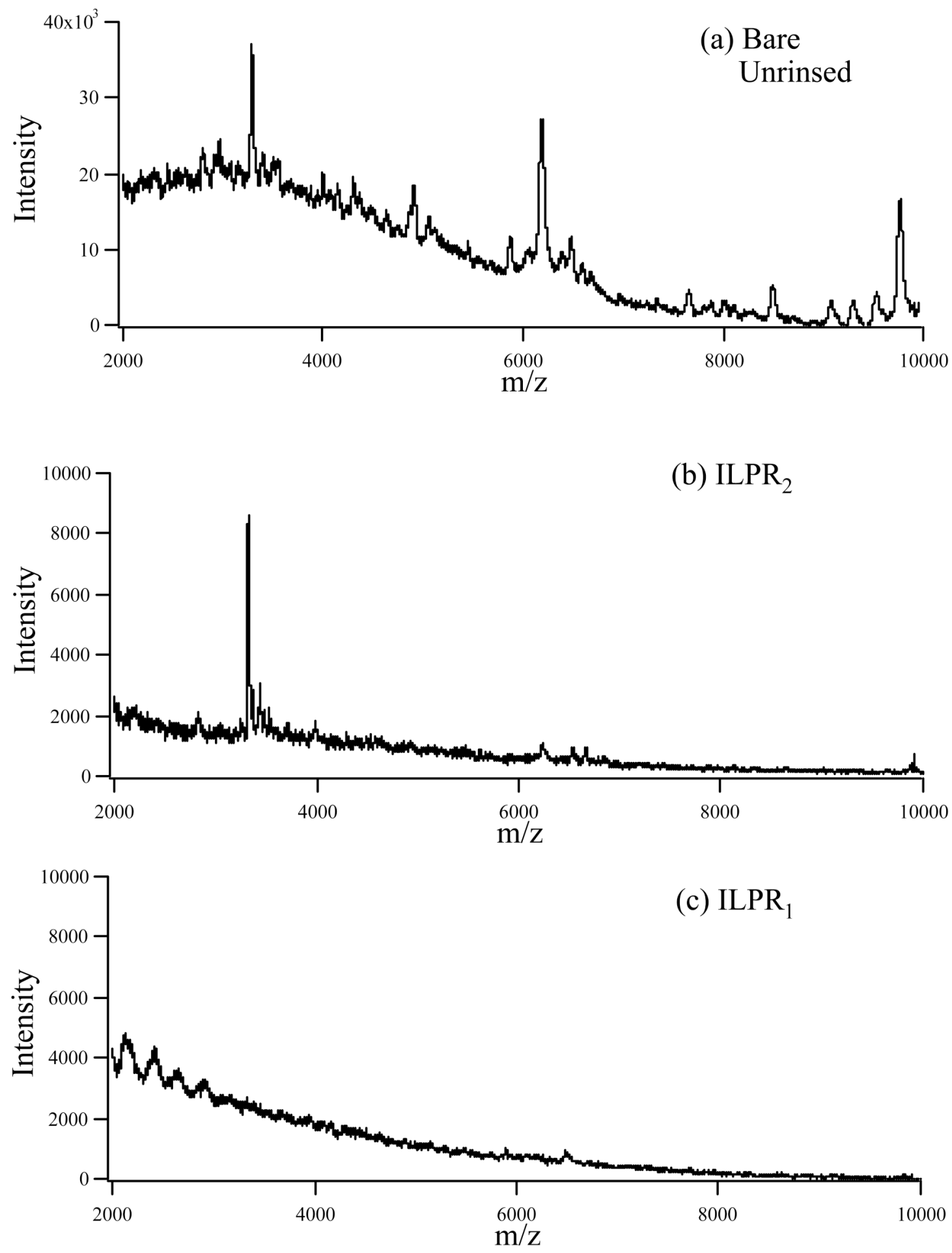

Figure 3.

Affinity MALDI-MS of HPNE on (a) bare fused silica, unrinsed, (b) ILPR , and (c) ILPR $_{1}$ surfaces. 

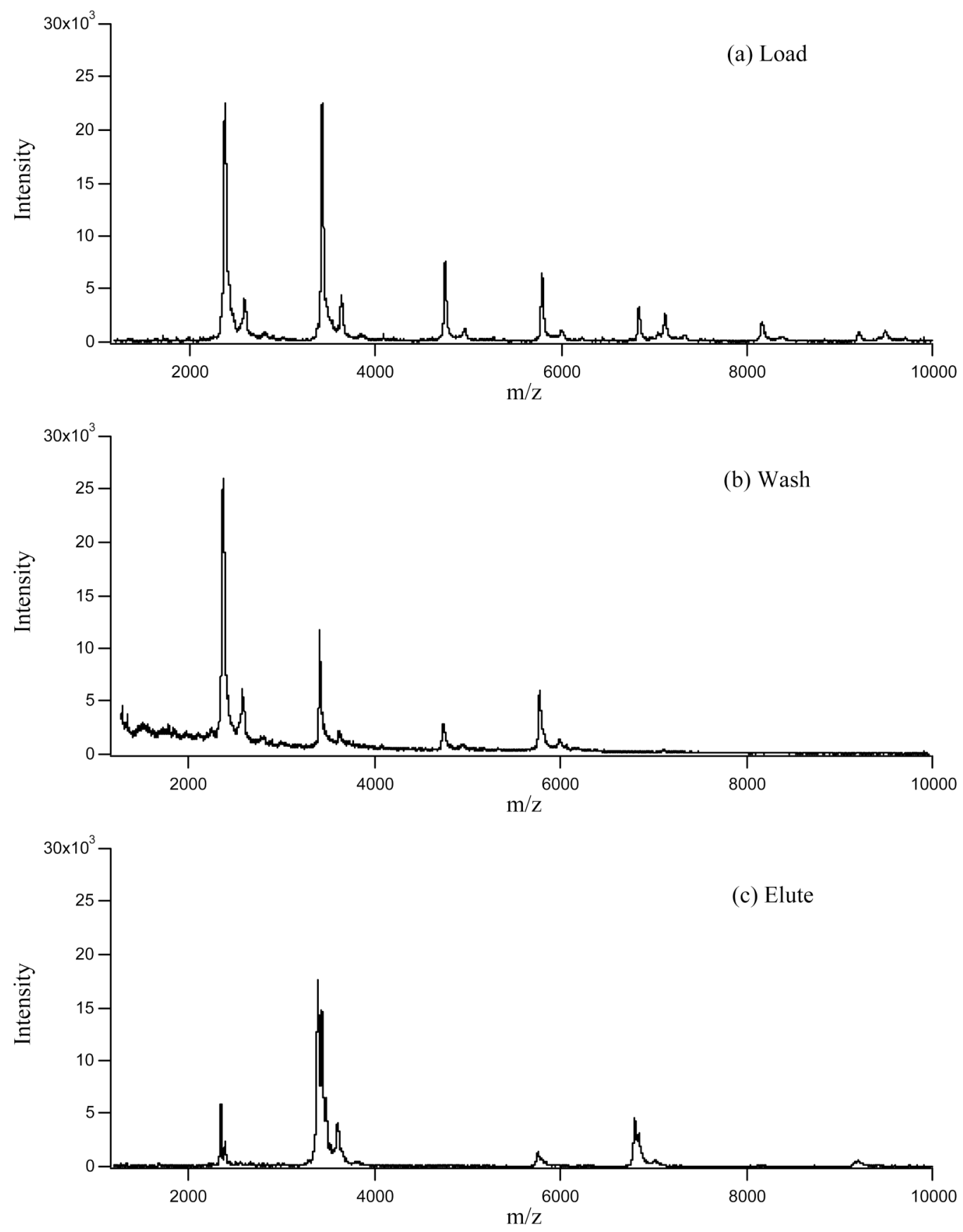

Figure 4.

MALDI mass spectra of collections from affinity capillary chromatography capture experiment of $500 \mu \mathrm{M}$ insulin incubated for $30 \mathrm{~min}$ with $500 \mu \mathrm{M}$ DTT before loading onto ILPR 2 capillary. 


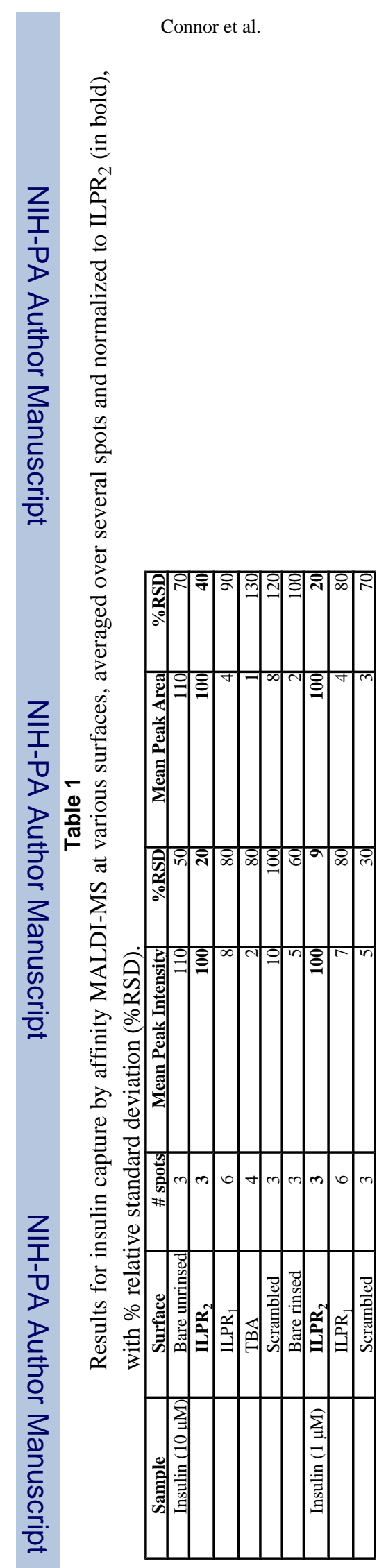

Page 13

$J$ Am Chem Soc. Author manuscript; available in PMC 2008 September 30. 